Retrospective Study

\title{
Palpation Versus Ultrasound-Guided Acromioclavicular Joint Intra-articular Corticosteroid Injections: A Retrospective Comparative Clinical Study
}

Ki Deok Park, MD, PhD1, Tai Kon Kim, MD², Jihae Lee, $\mathrm{MD}^{3}$, Woo Yong Lee, MD4, Jae Ki Ahn, MD', and Yongbum Park, MD

From: ${ }^{1}$ Department of Rehabilitation Medicine, Gachon University Gil Medical Center, Incheon, Republic of Korea; ${ }^{2}$ Department of Rehabilitation Medicine, Hanyang University College of Medicine, Seoul, Republic of Korea; ${ }^{3}$ Department of Radiology, Sanggye Paik Hospital, Inje University College of Medicine, Seoul, Republic of Korea; ${ }^{4}$ Department of Anesthesiology, Sanggye

Paik Hospital, Inje University College of Medicine, Seoul,

Republic of Korea; ${ }^{5}$ Department

of Rehabilitation Medicine,

Sanggye Paik Hospital, Inje University College of Medicine, Seoul, Republic of Korea

Address Correspondence: Yongbum Park, MD \#761-7 Sanggye 7 dong Nowon-gu, 139-707 Seoul, Republic of Korea

E-mail: swc328@naver.com

Disclaimer: There was no external funding in the preparation of this manuscript.

Conflict of interest: Each

author certifies that he or

she, or a member of his or her immediate family, has no commercial association (i.e., consultancies, stock ownership, equity interest, patent/licensing arrangements, etc.) that might

pose a conflict of interest in connection with the submitted manuscript.

Manuscript received: 10-14-2014

Revised manuscript received: 03-24-2015

Accepted for publication: 03-27-2015

Free full manuscript: www.painphysicianjournal.com
Background: Primary osteoarthritis $(\mathrm{OA})$ is the most common cause of pain arising from the acromioclavicular $(A C)$ joint. The true incidence is unknown because of differences in the criteria used to define arthritis in various studies. The proper diagnosis of AC joint OA requires a thorough physical examination, radiographic findings, and a diagnostic local anesthetic injection.

Objective: The goal of this study was to assess the effects and safety of ultrasound (US) versus palpation-guided acromioclavicular (AC) joint intra-articular (IA) corticosteroid injection for patients with osteoarthritis $(\mathrm{OA})$ of the $A C$ joint.

Study Design: Retrospective, compared clinical study.

Setting: University hospital outpatient pain clinic

Method: We retrospectively reviewed the charts of patients with AC joint degenerative OA who had undergone US or palpation-guided AC joint IA corticosteroid injection between January 2012 and December 2013 at our outpatient clinic. One hundred consecutive patients identified from chart review met inclusion criteria. Patients $(N=50)$ in US guide $A C$ joint IA steroid injection group were administered a mixture of $0.5 \%$ lidocaine $(1 \mathrm{~mL})$ with triamcinolone $(20 \mathrm{mg} / \mathrm{mL} ; 0.5 \mathrm{~mL})$ and radiographic contrast material $(0.5 \mathrm{~mL})$ and patients $(\mathrm{N}=50)$ in palpation-guided $A C$ joint IA steroid injection group were administered a mixture of $0.5 \%$ lidocaine $(1 \mathrm{~mL})$ with triamcinolone $(20 \mathrm{mg} / \mathrm{mL} ; 0.5 \mathrm{~mL})$ and $0.5 \mathrm{~mL}$ of radiographic contrast material. Results were measured using the Shoulder Pain and Disability Index (SPADI), Verbal Numeric pain Scale (VNS) at rest (VNSar), under local pressure (VNSIp), and the arm adduction test (VNSaat) at the joint space area before injections and at one, 3, and 6 months after the injections. Successful treatment occurred when patients obtained significant pain relief (as measured by $>50 \%$ improvement in the VNS score and 20 point improvement in the SPADI) at one, 3, and 6 months after the injections. Univariable analysis was performed to evaluate the relationship between possible outcome predictors and therapeutic effect by using a chi-square test. Logistic regression analysis was performed to assess whether injection method, injection accuracy, patients' age, gender, and duration of the disease were independent predictors of successful outcome.

Results: SPADI, VNSaat, VNSIp, and VNSar improved at one, 3, and 6 months after the injections in both groups. There was a statistically significantly greater improvement in the VNSIp score and SPDAl at 6 months and in the VNSaat score at 3 months and 6 months for US-guided group as compared with the palpation group. Successful treatment is defined as significant differences found between the groups or from the 3-month to 6-month outcomes. Multiple logistic regression and univariable analysis showed that the significant outcome predictors at 6-month follow-up was the injection accuracy.

Limitations: Limitations include the retrospective nature of the study, lack of evaluation of longterm effects, most of the injections were performed in patients with a BMI of less than $30 \mathrm{~kg} / \mathrm{m} 2$, and the treatment procedures were conducted by the same physician.

Conclusion: US-guided AC joint IA injection for the treatment of symptomatic AC joint OA resulted in better pain and functional status improvement than palpation-guided IA injection at the 6-month follow-up.

Key Words: Ultrasound, injection, acromioclavicular joint, steroid

Pain Physician 2015; 18:333-341 
P rimary osteoarthritis (OA) is the most common cause of pain arising from the acromioclavicular (AC) joint (1). The true incidence is unknown because of differences in the criteria used to define arthritis in various studies (2). Using morphologic criteria, De Palma et al (3) demonstrated degenerative changes by the fourth decade in the majority of AC joint specimens obtained from 151 patients. In one study of asymptomatic volunteers, findings indicative of $A C$ joint arthritis were present in $75 \%$ of shoulders (4). OA of the AC joint may be associated with other shoulder disorders and must be considered when evaluating any shoulder problem, especially rotator cuff impingement (2). The proper diagnosis of $A C$ joint $O A$ requires $a$ thorough physical examination, radiographic findings, and a diagnostic local anesthetic injection (5).

Intra-articular (IA) injections are often performed to treat AC joint OA. There have been several IA injection methods such as blind injection with anatomical landmark, fluoroscopically guided injection, ultrasound-guided (US) injection, and so on (6-8). There are several advantages to US, such as the absence of ionizing radiation and good availability. US-guided AC joint IA injection can be used to precisely localize the joint space and identify any possible effusions or swelling of the surrounding soft tissue $(9,10)$.

Whereas the diagnostic role of AC joint IA injection is widely accepted (2), the role of corticosteroid injection is less certain (11). According to a study on the long-term effects of IA corticosteroid injection in AC joint OA patients, there was continuous improvement for first year which was maintained for 5 years (12). Another study also stated that long-term effects were found in patients with immediate therapeutic effect (13). However, Jacob and Sallay (14), in an uncontrolled retrospective study, concluded that steroid IA injection offered only short-term relief and did not alter the progression of the disease. One of the reasons for conflicting results is that palpation-guided IA injection is less accurate than US-guided injection and therefore decreases reliability.

Previous studies on US-guided AC joint IA injection was limited to comparing the accuracy with a palpationguided approach for only 3 weeks $(7,9,15)$. Whereas this retrospective study compares treatment effect and safety for 6 months and analyzes the effectiveness of US-guided injection in AC joint OA patients.

The goal of this study is to assess the effects and safety of US- versus palpation-guided AC joint IA corticosteroid injection for patients with OA of the AC joint.

\section{Methods}

\section{Patient Selection}

This retrospective study was approved by the institutional review board of Sanggye Paik Hospital after all patients had been treated. This study was given a waiver of consent by the institutional review board. We retrospectively reviewed the charts of patients with $A C$ joint degenerative OA who had undergone US- or palpation-guided AC joint IA corticosteroid injection between January 2012 and December 2013 at our outpatient clinic. Diagnosis of AC joint degenerative OA were made based on history-taking, physical examination, plain x-ray evaluation, and musculoskeletal US findings. Patients who had shoulder pain localized to the $A C$ joint as well as tenderness to palpation over the $A C$ joint for at least 12 weeks were diagnosed with $A C$ joint degenerative OA, and were included in this study. In their history, the patients were questioned about pain duration, location, the increase in shoulder pain due to shoulder adduction, and presence of trauma. The most reliable provocative physical examination, the so-called crossbody adduction test, was performed. During the crossbody adduction test the arm on the affected side is elevated 90 degrees and the examiner then grasps the elbow and adducts the arm across the body. Reproduction of pain over the AC joint is suggestive of, although not specific for, an AC joint lesion $(2,5)$. AC joint disorder was suspected based on the history taken, if there was not presence of range of motion (ROM) limitation of the affected shoulder, no neurological deficit, and positive results in the crossbody adduction test. To evaluate the existing AC joint pathology and to exclude problems other than the AC joint causing shoulder pain, US and plain x-ray were used. Radiographic findings suggestive of degenerative OA include joint space narrowing, subchondral cysts, osteophytes, and subchondral sclerosis on anteroposterior views of the chest, anteroposterior and Grashey views of the shoulder, and Zanca view $(2,5)$. The exclusion criteria were as follows: US finding of a full thickness tear, a massive tear of the rotator cuff, shoulder joint plain radiographic finding of significant glenohumeral joint arthritis, systematic rheumatic disease, trauma to the shoulder, and a neurologic disorder, such as cervical radiculopathy or stroke.

Of the 148 AC joint OA patients' charts reviewed, a total of 100 patients who fulfilled the above criteria were identified. Patients $(N=50)$ in the US-guided AC joint IA steroid injection group were administered a 
Palpation Versus Ultrasound-Guided Acromioclavicular Joint Intra-articular Corticosteroid Injections

Table 1. General characteristics of the patients.

\begin{tabular}{|l|c|c|c||}
\hline & $\begin{array}{c}\text { Ultrasound-guided approach } \\
(\mathbf{n = 5 0})\end{array}$ & $\begin{array}{c}\text { Palpation-guided approach } \\
\text { (n= 50) }\end{array}$ & P value \\
\hline Age (year) & $57.8 \pm 8.4$ & $59.1 \pm 8.5$ & 0.375 \\
\hline Men & $11(22.0 \%)$ & $12(24.0 \%)$ & 0.812 \\
\hline Women & $39(78.0 \%)$ & $38(76.0 \%)$ & 0.832 \\
\hline Location (Right/Left) & $34(68 \%) / 16(32 \%)$ & $33(66 \%) / 17(34 \%)$ & 0.868 \\
\hline BMI (kg/m $\left.{ }^{2}\right)$ & $22.9 \pm 1.9$ & $22.8 \pm 2.1$ & 0.707 \\
\hline Duration (month) & $6.5 \pm 2.3$ & $6.6 \pm 2.2$ & \\
\hline
\end{tabular}

Values are in mean \pm standard deviation.

BMI: Body mass index

mixture of $0.5 \%$ lidocaine $(1 \mathrm{~mL})$ with triamcinolone $(20 \mathrm{mg} / \mathrm{mL} ; 0.5 \mathrm{~mL})$ and $0.5 \mathrm{~mL}$ of radiographic contrast material and patients $(\mathrm{N}=50)$ in palpation-guided $A C$ joint IA steroid injection group were administered a mixture of $0.5 \%$ lidocaine $(1 \mathrm{~mL})$ with triamcinolone (20 mg/mL; $0.5 \mathrm{~mL}$ ) and $0.5 \mathrm{~mL}$ of radiographic contrast material. Injection method was selected by patients after receiving an explanation about the pros and cons of the 2 methods, including costs. Patients in both groups had similar starting age, gender, body mass index (BMI), pathologic side, and duration (Table 1).

\section{US-guided IA Injection Techniques}

All US exams and US-guided procedures were conducted by a physiatrist (Y. Park) with more than 7 years of experience. All treatments were performed as an outpatient procedure. Accuvix XQ® (Medison, Seoul, Korea) with a linear probe at $6-12 \mathrm{MHz}$ was used as the US instrument. Patients were asked to sit on a chair without a backrest. The arm should be in the neutral position, as the deep joint space is the widest at this position (11). The injection site was disinfected with betadine and alcohol and covered with a sterilization wrap with pores to expose the applicable site only. The procedure was performed with aseptic gloves on. The probe was placed over the medial side of acromion in line with the clavicle. The AC joint can be visualized, with the capsule covering the 2 hyperechoic structures (acromion and clavicle) (11). We administered US-guided IA injections using the needle $(25 \mathrm{G}, 3.8 \mathrm{~cm})$ by an in-plain free-hand technique (Fig. 1A). All injections were made after ensuring that the needle tip was properly located in the IA space by US (Fig. 1B). In order to check the accuracy during injection, the direction of drug injection from the needle tip was checked with the color Doppler image (Fig. 1C).

\section{Palpation-guided AC Joint IA Injection}

The palpation-guided AC joint IA injections were performed by a physiatrist (Y. Park) with over 7 years of experience in musculoskeletal disorder. The medial acromion and lateral clavicle were palpated for patients while they were in the same posture with that of the US-guided IA injection and outlined with a marker. The shoulder was prepared using a broad enough area to permit palpation during the injection. The needle advanced in a lateral-to-medial direction $(2,7)$.

The needle passed between the bony margins of the acromion and clavicle, penetrating the AC joint capsule and superior ligaments (7). With gentle pressure, a pop or sudden change in resistance is felt as the needle penetrates the capsule and easy free flow confirms joint entry (7). If there was resistance to needle advancement or injection, needle repositioning was allowed. Afterward, the needle was moved slightly backward and the medication mixed with contrast agent was injected (Fig. 1D).

After an IA injection, an anteroposterior radiograph of the shoulder in a neutral position and a Zanca view of the $A C$ joint were taken to confirm that the IA injection had been performed correctly. A radiologist (J. Lee), who was blinded to the injection methods, judged all radiographic findings as either positive or negative. The result was considered positive when the nonionic contrast material was observed only within the AC joint and negative if the contrast material was visible in the extra-articular location (Fig. 2).

\section{Review of Clinical Data}

Using a retrospective study design, we reviewed collected clinical pain and function data on a consecutive sample of 100 patients with AC joint degenerative OA. We checked that a follow-up interview had 
been conducted in a hospital visit one, 3, and 6 months after injection, and checked that outcome measurements had been assessed by Shoulder Pain and Disability Index (SPADI), Verbal Numeric Pain Scale (VNS) at rest (VNSar), under local pressure (VNSIp), and the arm adduction test (VNSaat)



Fig 1. US-guided $A C$ joint IA injection.

Patients are in the seated position with the affected arm adducted at their side. A linear-array high-frequency transducer is placed vertically onto the skin, along the long axis of the clavicle, over the superior aspect of the AC joint area. The US shows the AC joint as a hypoechoic triangular-shaped gap between the acromion and the distal clavicle. (A) Needle approach and US transducer position for USguided acromioclavicular ( $A C$ ) joint injections. (B) The needle tip was observed within AC joint space. Arrow, needle shaft; arrowhead, AC joint. (C) Color Doppler image showing accurate intra-articular injection (white arrow). (D) Palpation-guided AC joint IA injection Patients are placed in the seated position with the affected arm resting comfortably at their side. To identify the $A C$ joint, palpate the clavicle distally to its termination at which point a slight depression will be felt at the joint articulation. Aseptic technique is followed. The medial acromion and lateral clavicle were palpated and the needle advanced in a lateralto-medial direction. The needle passed between the bony margins of the acromion and clavicle, penetrating the $A C$ joint capsule and superior ligaments. at the joint space area. When using VNS, the patients were asked to rate their pain on a scale from 0 to 10 where 0 and 10 represented "no pain" and "the worst pain possible," respectively, in whole numbers (16). SPADI was a self-administered assessment tool that measures pain and disability related to shoulder disease (17). It consists of 5 pain and 8 disability items each measured on a visual analogue scale. Pain and disability subscales are calculated as the mean of the corresponding items on a $0-100$ scale, the highest score indicating the most severe pain and disability (17). The total score is calculated as the sum of the pain and disability subscales. The patients with a reduction in the VNSaat of more than $50 \%$ and 20 points on the SPADI score after injection were classified as receiving effective treatment (17).

Independent variables such as injection method, IA or extra-articular injection, duration of symptoms, gender, and age were documented in the medical charts. Predictive variables were measured as follows: We classified the patient's age into 4 age groups, $40-49,50-59,60-69$, and $>70$. The duration of $A C$ joint $\mathrm{OA}$ was also treated as a potential predictive variable, and it was classified as acute or subacute, i.e., $<6$ months or chronic (> 6 months).

\subsection{Statistical Analysis}

Sample size was not calculated before the study as there was no currently published studies comparing these 2 techniques in terms of SPADI at 6 months' follow-up, which were considered as the primary outcomes. Therefore we determined that a sample size of $45-50$ patients per group had $90 \%$ power to detect a difference in the mean SPADI scores of $\geq 10$ if the standard deviation was $\leq 15$. 


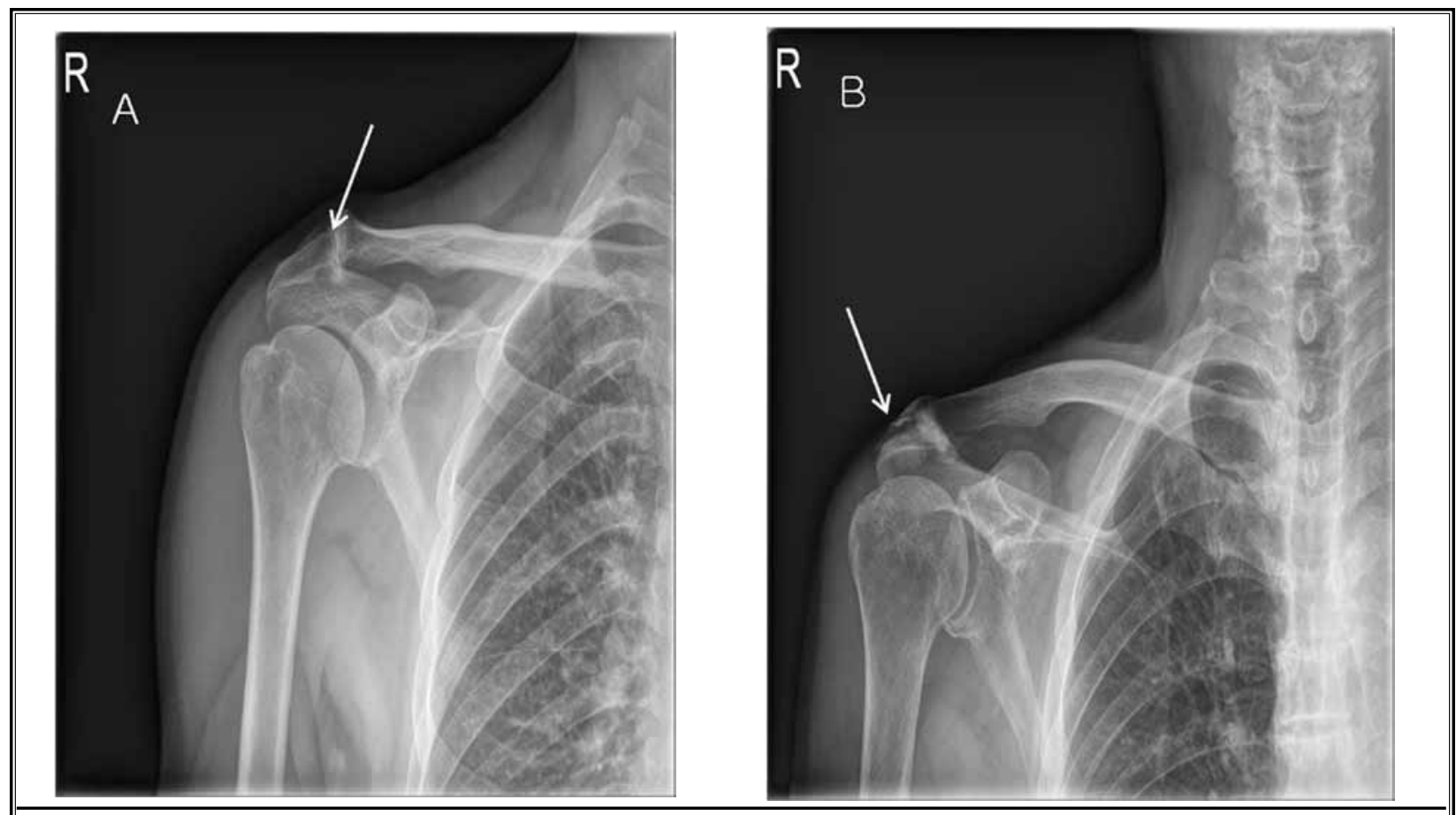

Fig 2. (A) Successful intra-articular injection (white arrow). (B) Failure of intra-articular injection with visible leakage (white arrow) of non-ionic contrast in the soft tissue.

The Pearson chi-square test and Mann-Whitney U method were used to compare the characteristics of 2 groups such as age, gender, BMI, pathologic side, and duration of the disease.

At each time point, all (at rest, under local pressure, and the arm adduction test) of the VNS and SPADI were compared by repeated measure analysis of variance (ANOVA), and the Bonferroni's correction was conducted for post-hoc comparison. The Chi-square test was used to test the differences in proportions. Fisher's exact test was used wherever the expected value was less than 5. Univariable analysis was performed to evaluate the relationship between possible outcome predictors and therapeutic effect by using the Chisquare test. Logistic regression analysis was performed to assess whether injection method, injection accuracy, patients' age, gender, or duration of the disease were independent predictors of successful outcome. Statistics were performed with SAS Enterprise Guide 4.1 (4.1.0.471) with significance level of $P<0.05$.

\section{Results}

Forty-eight of $50(96 \%)$ in the US-guided group were injected successfully as determined by the presence of contrast dye in the joint cavity by radiography.
However, only 31 of $50(60.5 \%)$ in the palpation group were successfully injected. A significantly higher success rate was observed in the US-guided group than in palpation-guided group $(P<0.05)$.

All (at rest, under local pressure, and the arm adduction test) of the VNS and SPADI after the injection improved significantly at one, 3, and 6 months in both groups (Table 2). There was a statistically significantly greater improvement in the VNSIp score and SPDAI at 6 months and in the VNSaat score at 3 months and 6 month for US-guided group as compared with the palpation group (Table 2). Fig. 3 illustrates the proportion of patients with a reduction on the VNSaat of $>$ $50 \%$ and SPADI of $>20$ points at one, 3 , and 6 months. Eighty-four percent in the US-guided group $(\mathrm{N}=42)$ and $72 \%$ in the palpation-guided group $(\mathrm{N}=36)$ showed improvement at one month, $68 \%$ in the US-guided group ( $\mathrm{N}=34$ ) and $54 \%$ in the palpation-guided group $(\mathrm{N}=27)$ at 3 months, and $58 \%$ in US-guided group ( $\mathrm{N}$ $=29)$ and $44 \%$ in palpation-guided group $(\mathrm{N}=22)$ at 6 months, respectively. Patients who dropped out due to treatment failure received additional treatment. At one month follow-up, there were 8 treatment failure patients in the US-guided injection group and 14 in the blind injection group. Six patients received reinjection 
Pain Physician: July/August 2015; 18:333-341

Table 2. Comparison of VNS at rest (VNSar), under local pressure (VNSlp), the arm adduction test (VNSaat) at the joint space, and SPADI by injection guidance from baseline to one, 3 , and 6 months.

\begin{tabular}{|c|c|c|c|c|c|}
\hline & & Baseline & One month & 3 months & 6 months \\
\hline \multirow{2}{*}{ VNSaat } & Ultrasound & $5.68 \pm 0.99$ & $2.64 \pm 0.78^{\star}$ & $2.50 \pm 0.71 \dagger^{*}$ & $2.20 \pm 0.98 \dagger^{*}$ \\
\hline & Palpation & $5.64 \pm 0.92$ & $2.94 \pm 0.87^{\star}$ & $2.85 \pm 0.78^{*}$ & $2.79 \pm 1.06^{*}$ \\
\hline \multirow{2}{*}{ VNSlp } & Ultrasound & $6.10 \pm 0.93$ & $2.82 \pm 0.69^{*}$ & $2.52 \pm 0.86^{*}$ & $2.29 \pm 1.06^{\star} \dagger$ \\
\hline & Palpation & $6.02 \pm 0.89$ & $2.94 \pm 0.87^{\star}$ & $2.94 \pm 0.89^{*}$ & $2.83 \pm 0.64^{*}$ \\
\hline \multirow{2}{*}{ VNSar } & Ultrasound & $5.16 \pm 0.79$ & $2.16 \pm 0.96^{*}$ & $2.45 \pm 0.83^{*}$ & $2.47 \pm 0.90^{*}$ \\
\hline & Palpation & $5.02 \pm 0.80$ & $2.18 \pm 0.80^{*}$ & $2.56 \pm 0.56^{*}$ & $2.29 \pm 0.75^{*}$ \\
\hline \multirow{2}{*}{ SPADI } & Ultrasound & $51.50 \pm 6.64$ & $23.88 \pm 4.57^{\star}$ & $25.71 \pm 5.01^{\star}$ & $27.44 \pm 6.07 \dagger^{\star}$ \\
\hline & Palpation & $52.88 \pm 7.96$ & $25.30 \pm 7.56^{*}$ & $28.12 \pm 6.75^{\star}$ & $30.63 \pm 5.59^{*}$ \\
\hline
\end{tabular}

Values are in mean \pm standard deviation. ${ }^{*} P<0.05$ : Comparison before and after the injection

$\dagger P<0.05$ : Comparison of ultrasound and palpation-guided approaches

Verbal Numeric pain Scale (VNS) at rest (VNSar), under local pressure (VNSlp) and the arm adduction test (VNSaat) at the joint space area, Shoulder Pain and Disability Index (SPADI)

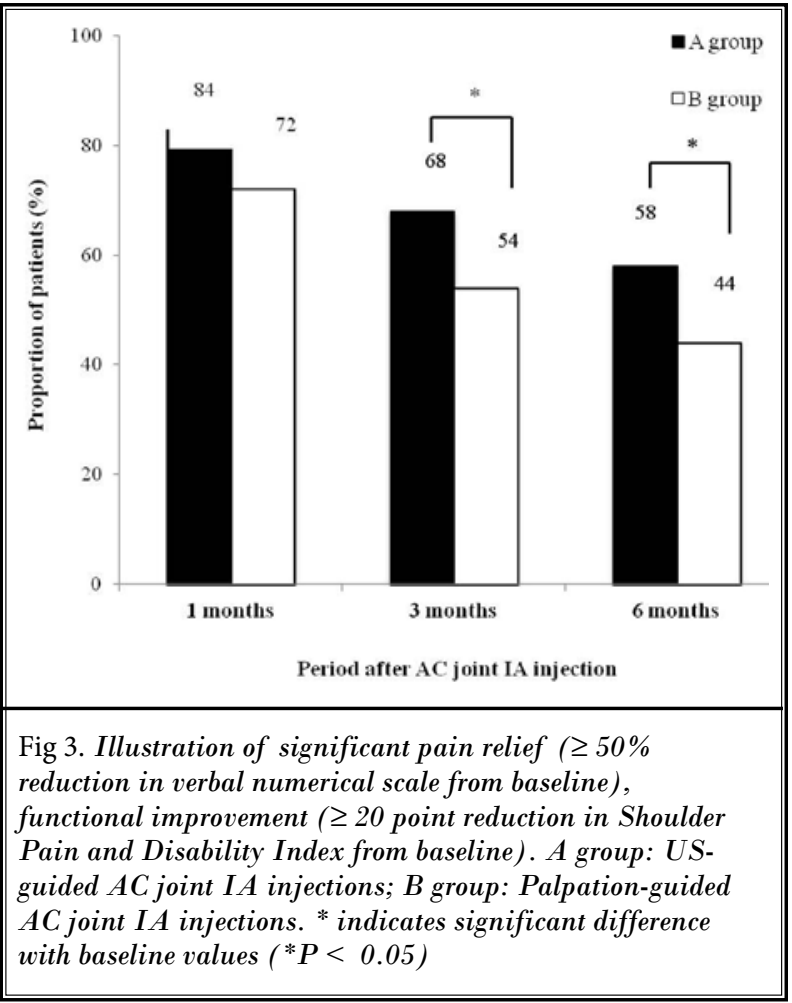

and 2 patients received additional non-steroidal antiinflammatory drugs (NSAIDs) in the US group. Eight patients received reinjection treatment, 3 patients received additional NSAIDs, and one patient received surgery (arthroscopic synovectomy) due to severe pain in the blind injection group. At 3 months follow-up, all the 8 treatment failure patients received reinjection in the US group. Seven patients received reinjection and 2 received additional NSAIDs in the blind injection group. At 6 month follow-up, all the treatment failure patients ( 5 in the US group and 5 in the blind injection group) received additional reinjection treatment. Successful outcomes were significant differences between the groups or from the 3-month to 6-month outcomes.

The whole study group was analyzed using a univariable analysis and logistic regression analysis to see if any predictors of an effective treatment to joint injection could be isolated. The model examined injection method, accuracy, patients' age, gender, and duration of the disease. Multiple logistic regression and univariate analysis showed that the significant outcome predictors at the 6-month follow-up was the injection accuracy (Tables 3 and 4).

Three patients in US-guided group and one patient in the palpation group complained of pain due to steroid-induced synovitis. Skin atrophy and depigmentation were observed in 2 patients in the palpation group, none in the US-guided group. There were no severe complications, such as septic arthritis or allergic reactions.

\section{Discussion}

$A C$ joint degenerative $O A$ is a common source of shoulder pain that is often neglected by clinicians and researchers because of the higher prevalence of rotator cuff pathology (2). Diagnostic uncertainty can be resolved through direct injection of local anesthetic into the AC joint $(2,7,18)$. Cadaveric studies on the accuracy of palpation-guided AC joint IA injections have reported rates of only $66 \%$ and in vivo studies have documented lower success rates from $40 \%$ to $57 \%$ $(6,19)$. Therefore image guidance has been recom- 
Palpation Versus Ultrasound-Guided Acromioclavicular Joint Intra-articular Corticosteroid Injections

mended to ensure accurate needle placement for diagnostic and therapeutic purposes as well as for safety (19). Fluoroscopically guided AC joint IA injections are well described and commonly used in clinical practice (2). However, fluoroscopy requires expensive and at times cumbersome equipment, exposes the operator and patient to ionizing radiation, and includes the additional risk of contrast agent reactions (8). US is probably more frequently available, much smaller, easy to handle, and does not need ionizing radiation (7,9-12). Also, US-guided interventions appear to be fast, less invasive, and decrease the risk of injury to neighboring soft tissue structures due to real-time monitoring during needle placement $(9-12,20)$.

US has recently been described as an alternative technique for guiding $A C$ joint IA injection (7,9-12). Sabeti-Aschraf et al (9) injected 60 AC joints in $60 \mathrm{ca}$ daveric specimens and reported a $95 \%$ accuracy rate. In addition, Peck et al (7) showed that with US guidance it is feasible to inject the AC joint with a high degree of accuracy by cadaveric study, and should be considered superior to palpation guidance. Our study is meaningful in that it was conducted in living subjects for the first time, showing the higher accuracy of $96.1 \%$ under US guidance compared to $57 \%$ with palpation guidance. Even in 2 failed cases in the US-guided group, contrast medium was in the AC joint as well as the surrounding tissue.

$\mathrm{OA}$ will respond to conservative treatment $(5,14,21)$. The best method of conservative treatment, however, is still controversial (13). The use of IA corticosteroid injections in the treatment of AC joint degenerative OA has conflicting, and mostly anecdotal, evidence in the literature (13). Hossain et al (13) reported that injection of corticosteroid into the $A C$ joint is an effective treatment for primary isolated $\mathrm{OA}$ and that this improvement of approximately $25 \%$ reduction in symptoms lasts at least 12 months. Jacob and Sallay (14), in an uncontrolled retrospective study, concluded that AC joint IA corticosteroid injection provides shortterm pain relief, but is not capable of stopping degenerative processes affecting the joint. van Riet et al (12) reported that only a minority of patients has sufficient pain relief from the injection at one month follow-up. It is considerable that the reason for such difference in results might be due to the low accuracy of palpationguided IA injection.

In previous studies on US-guided injection, SabetiAschraf et al (15) treated 10 patients by US guidance and 10 by a palpation technique. In both groups signifi-
Table 3. Univariate analysis for possible outcome predictors for injection effectiveness at 6 month follow-up.

\begin{tabular}{|c|c|c|c|}
\hline Characteristic & $\begin{array}{c}\text { Responders } \\
(\mathbf{N}=51)\end{array}$ & $\begin{array}{c}\text { Non- } \\
\text { Responders } \\
(\mathbf{N}=49)\end{array}$ & $P$ value \\
\hline \multicolumn{4}{|l|}{ Injection method } \\
\hline US (\%) & $29(56.9)$ & $21(42.9)$ & \\
\hline Palpation (\%) & $22(43.1)$ & $28(57.1)$ & 0.161 \\
\hline \multicolumn{4}{|l|}{ Gender } \\
\hline Men & $12(23.5)$ & $11(22.4)$ & \\
\hline Women & $39(76.5)$ & $38(77.6)$ & 0.898 \\
\hline \multicolumn{4}{|l|}{ Age } \\
\hline $40-49$ & $10(19.6)$ & $6(12.2)$ & \\
\hline $50-59$ & $22(43.1)$ & $24(49.0)$ & \\
\hline $60-69$ & $9(17.6)$ & $11(22.4)$ & \\
\hline 70 & $10(19.6)$ & $8(16.3)$ & 0.689 \\
\hline \multicolumn{4}{|l|}{ Duration } \\
\hline$<6$ month & $14(27.5)$ & $22(44.9)$ & \\
\hline 6 month & $37(72.5)$ & $27(55.1)$ & 0.069 \\
\hline \multicolumn{4}{|c|}{ Injection accuracy } \\
\hline Success & $51(100.0)$ & $29(59.2)$ & $<0.0001$ \\
\hline Fail & $0(0.0)$ & $20(40.8)$ & \\
\hline
\end{tabular}

Table 4. Possible outcome predictors for injection effectiveness at 6-month follow-up.

\begin{tabular}{|l|c|c|c|}
\hline Characteristic & OR & 95\% CI & P value \\
\hline Injection method & 1.758 & $0.796-3.880$ & 0.161 \\
\hline Gender & 0.941 & $0.370-2.390$ & 0.898 \\
\hline Age & 1.002 & $0.948-1.059$ & 0.954 \\
\hline Duration & 0.464 & $0.202-1.069$ & 0.069 \\
\hline Injection accuracy & 1.690 & $1.339-2.132$ & 0.001 \\
\hline
\end{tabular}

OR: Odds ratio; 95\% CI: 95\% Confidence interval

cant improvement in pain and function was obtained up to one week post injection. Function remained significantly improved until the last follow-up and did not differ between the 2 groups. Edelson et al (22) reported that subsequently, in the 15 patients who had isolated AC joint disease, VAS scores indicated significant improvement at final 6-month follow-up over preinjection values by an average of $5.5 \pm 1.4$. However both studies have limitations. Sabeti-Aschraf's study (15) has a short-term study period of 3 weeks and the other study (22) did not compare with a palpation-guided method. Throughout a 6-month study period, the US-guided method showed superior results to a palpation-guided 
method in VNSaat at 3months, and VNSIp, VNSaat, and SPDAl at 6 months. Also both methods showed a positive treatment effects at one, 3, and 6 months from the baseline.

We were able to confirm that injection accuracy can be used as a strong factor for predicting therapeutic effect after 6 months following injection. Nam et al (23) demonstrated that there was a significantly greater improvement in the clinical outcomes in the accurate injection group $(\mathrm{N}=50)$ compared with the inaccurate injection group $(\mathrm{N}=7)$ as assessed by patients with distal radioulnar joint pain at one, 3, and 6 months after injection. Cunnington et al (24) reported that accurate injections led to greater improvement in joint function as determined by VAS scores at 6 weeks, when compared with inaccurate injections. Thus, accurate injection into IA space ensures effective treatment outcomes.

Corticosteroid injections can cause temporary increases in pain, skin atrophy, depigmentation, and septic arthritis as well as deleterious effects on intraarticular cartilage or tendon degeneration and even tendon ruptures (25). Skin atrophy and depigmentation were the second most frequently reported side effects (4\%). Although most side effects are rare and temporary, skin atrophy and depigmentation can be permanent and should be explained to the patient before performing an injection. In this study, there were 2 cases of skin atrophy and depigmentation in the palpation-guided group, and none in US-guided group. Although there was no statistical significance, all of cases were by extracapsular placement of the injected mixture. So, accurate intra-articular injection may be essential to ensure the safety of the procedure, and this can be provided by the use of modalities such as US.

There have been 2 methods for US-guided AC joint injection presented until now and both methods use free-hand techniques. Sabeti-Aschraf's study $(9,15)$ and Edelson et al (22) both used the long-axis outof-plain (OOP) method; Peck et al (7) used the long axis in plain (IP) method. The OOP method involves inserting the needle so that it crosses the plane of imaging near the target. With this method, the target is typically centered within the field of view and the depth is noted. With the IP method, the needle can be inserted within the plane of imaging to visualize the entire shaft and the tip. Therefore, the imaged needle path should be maximized by placing the target on the side of the imaging field of view away from the approaching needle (26). We have used the IP method as in the Peck et al (7) study. However, there are pros and cons compared to the OOP method. Critics of the IP method are concerned that this approach is timeconsuming and that partial lineups of the needle and the transducer create a false sense of security (27). Another potential disadvantage of the IP method is the associated reverberation created from the long axis of the needle shaft that may impair detection of structures below the imaged procedure needle shaft (27). The IP method requires longer needle insertion paths than the OOP method and can therefore cause more discomfort for the patient (28). Critics of the OOP method are concerned about complications due to the lack of needle tip visibility during the procedure. Finding an echogenic dot for the OOP method within a bright background can be difficult (27). In addition, the OOP method poses difficulty in accurately following the procedure needle to select the target or lack of assurance to about whether the hyperechoic dot seen in the US image is an approximation of the procedure needle tip or needle shaft. Studies comparing the 2 US-guided methods will be necessary in the future.

The present study has several limitations. First, it is a retrospective study. For example, we could not determine socioeconomic status of patients which would be a variable to the effectiveness of injections. Patients in this study were $75 \%$ female. This could be from selection bias. And so a randomized prospective study including a large number of patients is warranted. Second, longterm effects should be evaluated in the future based on the results of the mid-term effects. Third, most of the injections were performed in patients with a BMI of less than $30 \mathrm{~kg} / \mathrm{m} 2$. The degree of difficulty in performing the US-guided injection could potentially be accentuated by a patient's high body mass index (BMI), which increases the attenuation and depth of the injection site. Needle visualization decreases because of the steeper angle that is required for a deeper injection. Further study including obese patients could clarify this limitation. Fourth, the treatment procedures were conducted by the same physician. The study results may have reflected the experience of one practitioner which may have limited generalization of the study.

\section{Conclusion}

Corticosteroid IA injection in patients with AC joint OA results in significant alleviation of symptoms and improvement in functional status that is sustained at 6 months follow-up in a high proportion of patients. There was a statistically significant greater improve- 
ment in pain and functional status at 6-month followups for the US-guided group as compared with the palpation group. Also this study finds an association between treatment outcome and injection accuracy. In conclusion, the US-guided method, which has higher accuracy and outstanding treatment effect, should be used for the corticosteroid injection treatment of symptomatic AC joint OA.

\section{References}

1. Henry $\mathrm{MH}$, Liu SH, Loffredo AJ. Arthroscopic management of the acromioclavicular joint disorder. A review. Clin Orthop Relat Res 1995; 316:276-283.

2. Shaffer BS. Painful conditions of the acromioclavicular joint. J Am Acad Orthop Surg 1999; 7:176-188.

3. DePalma AF, Callery G, Bennett GA. Variational anatomy and degenerative lesions of the shoulder joint. Instr Course Lect 1949; 6:255-281.

4. Needell SD, Zlatkin MB, Sher JS, Murphy BJ, Uribe JW. MR imaging of the rotator cuff: Peritendinous and bone abnormalities in an asymptomatic population. AJR Am J Roentgenol 1996; 166:863-867.

5. Buttaci CJ, Stitik TP, Yonclas PP, Foye PM. Osteoarthritis of the acromioclavicular joint: A review of anatomy, biomechanics, diagnosis, and treatment. Am J Phys Med Rehabil 2004; 83:791-797.

6. Partington PF, Broome GH. Diagnostic injection around the shoulder: Hit and miss? A cadaveric study of injection accuracy. J Shoulder Elbow Surg 1998; 7:147-150.

7. Peck E, Lai JK, Pawlina W, Smith J. Accuracy of ultrasound-guided versus palpation-guided acromioclavicular joint injections: A cadaveric study. PMER 2010; 2:817-821.

8. Pichler W, Weinberg AM, Grechenig S, Tesch NP, Heidari N, Grechenig W. Intra-articular injection of the acromioclavicular joint. J Bone Joint Surg $\mathrm{Br} 2009$; 91:1638-1640.

9. Sabeti-Aschraf $M$, Lemmerhofer B, Lang S, Schmidt M, Funovics PT, Ziai P, Frenzel S, Kolb A, Graf A, SchuellerWeidekamm C. Ultrasound guidance improves the accuracy of the acromioclavicular joint infiltration: A prospective randomized study. Knee Surg Sports Traumatol Arthrosc 2011; 19:292-295.

10. Collins JM, Smithuis R, Rutten MJ. USguided injection of the upper and lower extremity joints. Eur J Radiol 2012;
81:2759-2770.

11. Peng PW, Cheng P. Ultrasound-guided interventional procedures in pain medicine: A review of anatomy, sonoanatomy, and procedures. Part III: shoulder. Reg Anesth Pain Med 2011; 36:592-605.

12. van Riet RP, Goehre T, Bell SN. The long term effect of an intra-articular injection of corticosteroids in the acromioclavicular joint. J Shoulder Elbow Surg 2012; 21:376-379.

13. Hossain S1, Jacobs LG, Hashmi R. The long-term effectiveness of steroid injections in primary acromioclavicular joint arthritis: A five-year prospective study. J Shoulder Elbow Surg 2008; 17:535-538.

14. Jacob AK, Sallay PI. Therapeutic efficacy of corticosteroid injectionsin the acromioclavicular joint. Biomed Sci Instrum 1997; 34:380-385.

15. Sabeti-Aschraf M, Ochsner A, SchuellerWeidekamm C, Schmidt M, Funovics PT, V Skrbensky G, Goll A, Schatz KD. The infiltration of the $A C$ joint performed by one specialist: Ultrasound versus palpation a prospective randomized pilot study. Eur J Radiol 2010; 75:e37-e40.

16. Hartrick CT, Kovan JP, Shapiro S. The numeric rating scale for clinical pain measurement: A ratio measure? Pain Pract 2003; 3:310-316.

17. Roach KE, Budiman-Mak E, Songsiridej N, Lertratanakul. Development of a shoulder pain and disability index. Arthritis Care Res 1991; 4:143-149.

18. Tallia AF, Cardone DA. Diagnostic and therapeutic injection of the shoulder region. Am Fam Physician 2003; 67:1271-1278.

19. Bain Gl, van Riet RP, Gooi C, Ashwood N. The long-term efficacy of corticosteroid injection into the acromioclavicular joint using a dynamic fluoroscopic method. Int ] Shoulder Surg 2007; 1:104-107.

20. Rutten MJ, Collins JM, Maresh BJ Smeets $\mathrm{JH}$, Janssen CM, Kiemeney LA Jager $\mathrm{G}$. Glenohumeral joint injection: A comparative study of ultrasound and fluoroscopically guided techniques before MR arthrography. Eur Radiol 2009; 19:722-730.

21. Strobel K, Pfirrmann CW, Zanetti M, Nagy L, Hodler J. MRI features of the acromioclavicular joint that predict pain relief from intraarticular injection. Am J Roentgenol 2003; 181:755-760.

22. Edelson G, Saffuri H, Obid E, Lipovsky $E$, Ben-David D. Successful injection of the acromioclavicular joint with use of ultrasound: Anatomy, technique, and follow-up. J Shoulder Elbow Surg 2014; 23:e243-e250.

23. Nam SH, Kim J, Lee JH, Ahn J, Kim YJ, Park Y. Palpation versus ultrasoundguided corticosteroid injections and short-term effect in the distal radioulnar joint disorder: A randomized, prospective single-blinded study. Clin Rheumatol 2014; 12:1807-1814.

24. Cunnington J, Marshall N, Hide G, Bracewell C, Isaacs J, Platt P, Kane D. A randomized, double-blind, controlled study of ultrasound-guided corticosteroid injection into the joint of patients with inflammatory arthritis. Arthritis Rheum 2010; 62:1862-1869.

25. Gaujoux-Viala C, Dougados M, Gossec L. Efficacy and safety of steroid injections for shoulder and elbow tendonitis: A meta-analysis of randomised controlled trials. Ann Rheum Dis 2009; 68:1843-1849.

26. Gray AT. Ultrasound-guided regional anesthesia: Current state of the art. Anesthesiology 2006; 104:368-373.

27. Souzdalnitski D, Lerman I, Halaszynski TM. How to improve needle visibility. In: Narouze SN (ed). Atlas of Ultrasoundguided Procedures in Interventional Pain Management. 1st ed. Springer, New York, 2011, pp 35-75.

28. Marhofer P, Greher M, Kapral S. Ultrasound guidance in regional anaesthesia. $\mathrm{Br}$ J Anaesth 2005; 94:7-17. 
